Balasa D. ${ }^{1}$, Ciufu Carmen ${ }^{2}$, Baz R. ${ }^{3}$, Hancu Anca ${ }^{4}$

\title{
Compressive Lumbar Disc Hernia Spontaneously Disapeared In A Few Years - Case Report
}

${ }^{1}$ Compressive Lumbar Disc Hernia Spontaneously Disapeared In A Few Years, Case Report

${ }^{2}$ Mediconst, Radiological Clinic, Constanta

${ }^{3}$ Pozimed, Radiological Clinic, Constanta

${ }^{4}$ Department Neurology, Clinical Emergency County Hospital Constanta

\begin{abstract}
We present a patient with left compressive L5S1 lumbar disc hernia in lateral reces and foramina revealed by high intensity pain (Visual Autologus Scale 7/10) and paresthesias on the left S1 dermatoma for 5 months. He refused surgery and followed conservative treatment with very good results after 6 months. The radicular S1 pain became of a small intensity (Visual Autologus Scale 2-3/10) and intermitent and after one year it completely disapeared. After 4 years he repeated the lumbar magnetic resonance imaging which proved the disapearance of the L5-S1 lumbar disc hernia.
\end{abstract}

Keywords: Lumbar disc hernia, disapeared
spontaneously

Daniel Balasa

Clinical Emergency County Hospital Constanta, Department Neurosurgery

Tomis Boulevard, 145, Constanta, Romania

email : daniel_balasa@yahoo.com

phone: $+407 \overline{4} 4682613$

\section{Introduction}

Lumbar disc hernia (LDH) is a common cause of low back pain and/or radicular crural pain [1]. According to Ushewokunze [2] 5\% of the patients with low back pain have LDH. Motor and/or sensory deficits are present in $50-90 \%$ of the patients LDH [2]. According to Weinstein [3], for the patients with algoparesthesias on crural dermatomas as symptomatology is little evidence available that surgery is better than conservative treatment. There are some articles in the medical literature who present the cases with spontaneous regression of LDH treated conservative[4,5]. We present a case of a patient with compressive L5-S1 LDH (proved on magnetic resonance imaging-MRI) which also had clinical criteria for surgery (unbearable radicular pain S1 left ) but choosed conservative treatment being afraid to be operated.

\section{Ease report}

We present the case of a 55 years old male 
patient who acused (in 2012) intense pain (Visual Autologus Scale -VAS- 7/10) and paresthesias on the left S1 dermatoma for 5 months. Lumbar vertebral MRI revealed a compressive left L5-S1 LDH in the lateral reces and foramina.

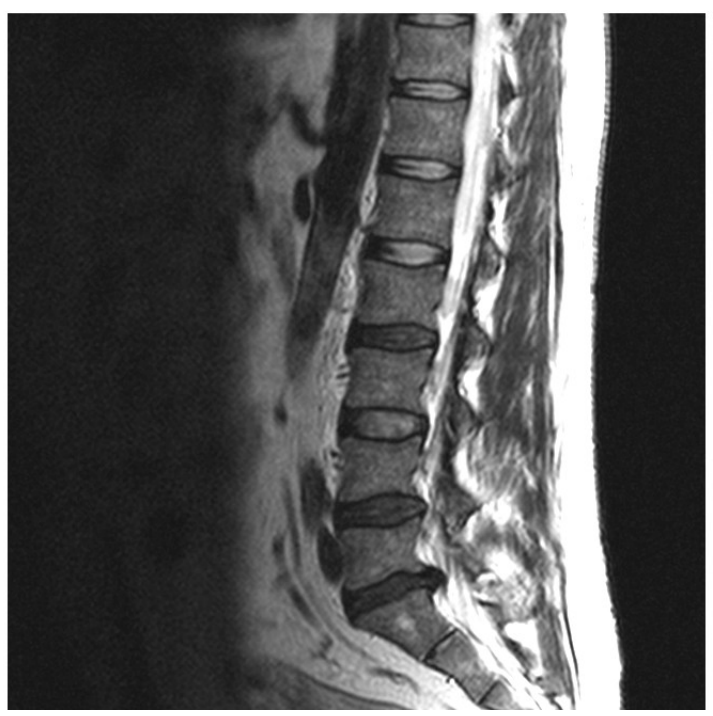

Figure 1. MRI sagital T2. Left L5-S1 LDH (protrusion type) compresive in lateral reces (white and blue arrow). PLL is intact in L5-S1 left posterior disc space (black double arrow)

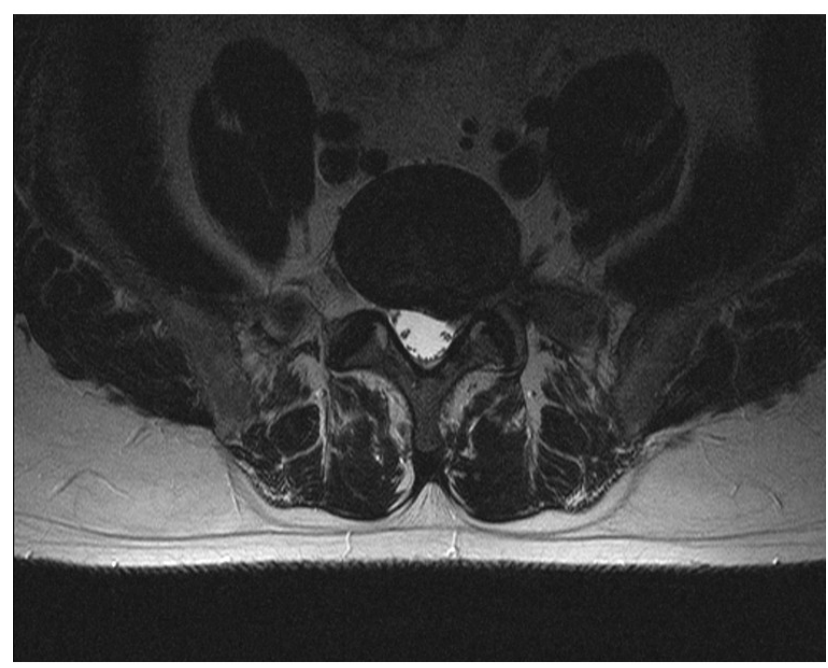

Figure 2. MRI axial T2. Left L5-S1 LDH compresive in lateral reces and left foramina (white and blue arrow)

He refused surgery and followed conservative treatment (acupuncture, analgetics, short term nonsteroid antiinflamatory treatment, physiotherapy). After 6 months the radicular S1 pain became of a small intensity (VAS 2-3/10) and intermitent and after one year it completely disapeared. After 4 years (2016) the new MRI proved disapearence of the L5S1 LDH.

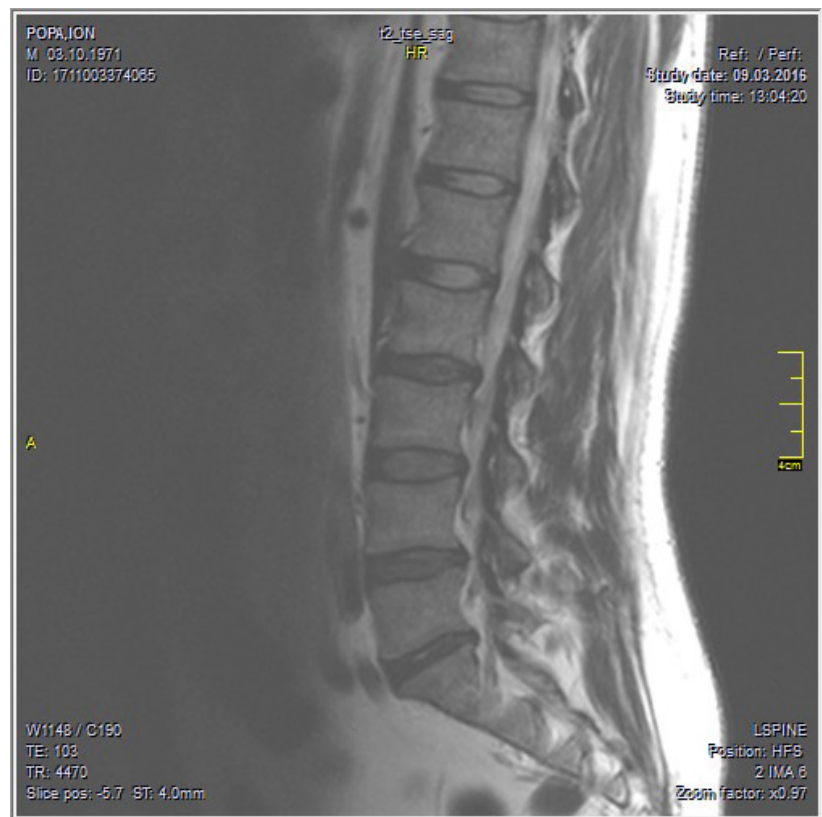

Figure 3. MRI sagital T2. L5-S1 disc. Normal aspect (white and blue arrow)

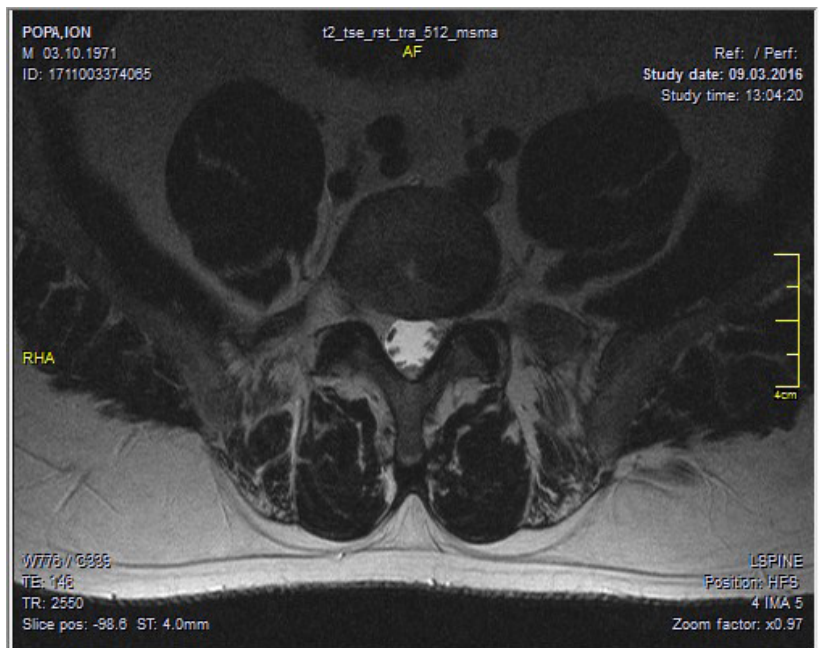

Figure 4. MRI axial T2. L5-S1 disc. Normal aspect (white and blue arrow) 


\section{Discussion}

According to Ushewokunze [2] 5\% of the patients with low back pain have LDH. Motor and/or sensory deficits are present in $50-90 \%$ of the patients with LDH [2]. According to Weinstein [3], for the patients with algoparesthesias on crural dermatomas as symptomatology is little evidence available that surgery is better than conservative treatment. When the symptoms of the patients are only pain and paresthesias the surgical treatment is recomended only after a failure of 6 months of conservative treatment (according to the recomendations of the Comitee of AO Spine Society).

The management of LDH may involve conservative and operative treatments [1]. Conservative treatment includes continued activity, analgesia, physiotherapy [1]. Surgical treatment may include standard discectomy, microdiscectomy, percutaneous discectomy, laser discectomy1. According to Martinez-Quinones [6] clinical criterias who require surgical treatment are:

- Cauda equina syndrome

- Progressive or serious motor deficit

- Unbearable pain (VAS 7-10/10)

The introduction of magnetic resonance imaging made possible accurate diagnosis, prognosis and surgical strategy of the patients with LDH [5]

According to Ito [7], the mechanism proposed as an explanation for reduction in size of the LDH was the absorptive process that relies on neovascularisation and infiltration by inflammatory cells as macrophages, granulocytes and lymphocytes [7].

According to Martinez-Quinones [6] the factors who may contribute to the regression of the $\mathrm{LDH}$, other than Ito postulated, are:

- Dehydration mechanism

- Pulsion of the cerebro-spinal fluid against the herniated disc portion.

The clue of this case is to carefully monitor the patient's pain (for the patients who had pain and paresthesias ) intensity. Keep in mind the AO
Spine Comitee recommendation about six months of conservative period for this kind of patients. If after this period of time, the treatment becomes eficient ( the pain intensity decrease around or more than $50 \%$ ) this is a sign that the surgery and the intra/ perioperative surgical risks can be avoided. The evolution of this case is a clear demonstration of this principle.

\section{Bonclusion}

We present a patient who acused intense pain (VAS 7/10) and paresthesias on the left S1 dermatoma for 5 months. Lumbar vertebral MRI revealed a compressive left L5-S1 LDH in the lateral reces and foramina. He refused surgery and followed conservative treatment with very good result after 6 months of conservative treatment. After 4 years (2016) the new MRI proved the disapearence of the L5-S1 left LDH.

\section{References}

1. Deyo, R.A. \& Weinstein, J.N. (2001). Low back pain. $N$ Engl J Med. 344(5), 363-370. doi: 10.1056/NEJM200102013440508

2. Ushewokunze, S., Abbas, N., Dardis, R. \& Killeen, I. (2008). Spontaneously disappearing lumbar disc protrusion. Br J Gen Pract. 58(554), 646-647. doi: 10.3399/bjgp08X341968

3. Weinstein, J.N., Lurie, J.D., Tosteson, T.D., Skinner, J.S., Hanscom, B., Tosteson, A.N., Herkowitz, H., Fischgrund, J., Cammisa, F.P., Albert, T. \& Deyo, R.A. (2006). Surgical vs nonoperative treatment for lumbar disk herniation: the Spine Patient Outcomes Research Trial 
(SPORT) observational cohort. JAMA. 296(20), 2451-2459. doi: 10.1001/jama.296.20.245

4. Ellenberg, M., Reina, N., Ross, M., Chodoroff, G., Honet, J.C. \& Gross, N. (1989). Regression of herniated nucleus pulposus: two patients with lumbar radiculopathy. Arch Phys Med Rehabil. 70(12), 842-844.

5. Keskil, S., Ayberk, G., Evliyaoglu, C., Kizartici, T., Yucel, E. \& Anbarci, H. (2004). Spontaneous resolution of "protruded" lumbar discs. Minim Invasive Neurosurg. 47(4), 226-229. doi: $10.1055 / \mathrm{s}-2004-818517$

6. Martinez-Quinones, J.V., Aso-Escario, J., Consolini, F. \& Arregui-Calvo, R. (2010). [Spontaneous regression from intervertebral disc herniation. Propos of a series of 37 cases]. Neurocirugia (Astur). 21(2), 108-117.

7. Ito, T., Yamada, M., Ikuta, F., Fukuda, T., Hoshi, S.I., Kawaji, Y., Uchiyama, S., Homma, T. \& Takahashi, H.E. (1996). Histologic evidence of absorption of sequestration-type herniated disc. Spine (Phila Pa 1976). 21(2), 230-234. 